\title{
Comparison of Postoperative Hepatic Function between Laparoscopic and Open Cholecystectomy
}

\author{
Sefik Hasukic ${ }^{a}$ Dragutin Kosuta ${ }^{b}$ Kasim Muminhodzic ${ }^{c}$ \\ University Departments of a Surgery and ${ }^{\mathrm{c}}$ Gastroenterology, University Clinical Center, \\ Faculty of Medicine, University of Tuzla, Tuzla, Bosnia-Herzegovina; ${ }^{b}$ University Department of Surgery, \\ 'Sveti Duh' General Hospital, Zagreb, Croatia
}

\section{Key Words}

Laparoscopic cholecystectomy · Open

cholecystectomy $\cdot$ Liver function tests $\cdot$ Hepatic

function

\begin{abstract}
Objective: In this prospective study, we evaluated the effects of pneumoperitoneum on hepatic function during laparoscopic (LC) and open cholecystectomy (OC). Subjects and Methods: One hundred patients who underwent LC $(n=50)$ or OC $(n=50)$ were included in the study. The groups were similar in age, sex, weight and height. Following liver function tests (total bilirubin; $\gamma$-glutamyltransferase, GGT; alkaline phosphatase, ALP), aspartate aminotransferase (AST), alanine aminotransferase (ALT) and lactate dehydrogenase (LDH) were obtained preoperatively and at 24 and $48 \mathrm{~h}$ postoperatively. Similar anesthesiologic protocol was used for both LC and OC. During LC, the intra-abdominal pressure was maintained within the conventional range of 12-14 mm Hg. Results: Total bilirubin, ALP, GGT and LDH levels remained unchanged from baseline in both groups without significant difference between them. A higher number of patients had increased values of ALT (26/50 vs. 5/50) and AST (23/50 vs. 6/50) in LC compared to OC group. Al-
\end{abstract}

though the difference was statistically significant ( $p<$ 0.000 for ALT and $p=0.0004$ for AST) the increased level decreased at 48 compared to 24 h. Conclusion: The results indicate that $L C$ is associated with transient elevation of ALT and AST. The disturbances in the function of the liver after LC are self-limited and not associated with any morbidity in patients with a normal liver function.

Copyright (C) 2005 S. Karger AG, Basel

\section{Introduction}

Laparoscopic cholecystectomy (LC) is 'the gold standard' in treating symptomatic gallstones. Its advantages include less patient discomfort, better cosmetic results, shorter hospital stay and shorter interval to return to work [1]. It is now commonly accepted that pneumoperitoneum causes intraoperative cardiovascular, respiratory, hepatic and renal adverse effects [2-4]. Postoperative changes in liver function tests after abdominal surgery and open cholecystectomy (OC) have been adequately described in the literature [4-9]. The disturbance can be caused by anesthetic drugs (halothane, nitrous oxide) or by circulatory failure during operation. However, postoperative changes in liver function after LC have not been adequately studied. It has been reported that hemo-

\section{KARGER}

Fax +4161306 1234 E-Mail karger@karger.ch www.karger.com
Sefik Hasukic, MD

University Clinical Center, Department of Surgery Trnovac b.b.

BA-75 000 Tuzla (Bosnia-Herzegovina)

Tel. +387 35238 357, Fax +387 35250 474, E-Mail shasukic@bih.net.ba 
dynamic disturbances in the abdomen reduce portal venous flow and cause physiological alterations in hepatic function [10, 11]. Middle hepatic venous blood flow, continuously measured by transesophageal echocardiography, is decreased significantly during a period of high intraperitoneal pressure in elderly patients undergoing LC [11]. Consequently, elevation in the levels of liver enzymes following LC is an obvious cause of apprehension to the surgeon concerned about the integrity of the bile ducts $[7,10]$. It has been shown that the duration and level of intra-abdominal pressure are responsible for changes in hepatic function during LC [7]. Halevy et al. [6] reported a significant increase in postoperative liver enzymes (alanine aminotransferase, ALT; aspartate aminotransferase, AST) in up to $80 \%$ of patients undergoing LC. Hence the present study was designed to determine and compare changes in liver function tests following LC and OC.

\section{Subjects and Methods}

From January 2002 to October 2002, 146 consecutive patients with symptomatic gallstones eligible for cholecystectomy were initially enrolled in this study. Local ethics committee approval was obtained. Gallbladder disease was diagnosed on the basis of clinical history and ultrasound examination. Randomization to LC or OC was performed immediately before the operation. Patients were either ASA grade I or II and those with higher ASA grades were excluded from the study. Only the patients with preoperatively normal liver function tests were included in the study. Other inclusion criteria were age (18-60 years), regular body weight and height, no previous abdominal surgery, no gallstone-related complications, absence of cardiovascular and hepatic dysfunction, no conversion to $\mathrm{OC}$, no postoperative bile duct complications such as injury, obstruction or infection. Of the 146 patients, 100 met the inclusion criteria and were randomized into LC $(n=50)$ and OC $(n=50)$ patients. The remaining 46 patients were excluded for various reasons including 2 patients who refused randomization. Both LC and OC patients were similar in age, sex, height, and weight. LC was performed using a three-trocar technique, with patients in reverse Trendelenburg's position, insufflating the abdomen with $\mathrm{CO}_{2}$ using an automatic insufflator set at 3-5 liters/min, to a maximum pressure of $14 \mathrm{~mm} \mathrm{Hg}$. A subcostal incision was performed for OC. Monopolar electrocautery was used in both the LC and OC patients to dissect the gallbladder from the liver bed. A standardized anesthetic technique was used for all patients. Anesthesia was induced and maintained with infusion of propofol and alfentanyl. Minute ventilation was adjusted to maintain the end-tidal $\mathrm{CO}_{2}$ at $4.5 \mathrm{kPa}$. All the patients received 1 liter of Hartmann's solution during the procedure. All the patients were intubated nasogastrically; the stomach was emptied during and at the end of surgery.

The following liver function tests were performed preoperatively and 24 and $48 \mathrm{~h}$ postoperatively: total bilirubin, aminotransferase (ALT, AST), alkaline phosphatase (ALP), $\gamma$-glutamyltranspeptidase (GGT) and lactate dehydrogenase (LDH). All data were ex-
Table 1. Patient clinical data

\begin{tabular}{lcll}
\hline Clinical data & LC & OC & $\mathrm{p}$ \\
\hline Patients & 50 & 50 & \\
Sex ratio, m/f & $4 / 46$ & $5 / 45$ & NS \\
Mean age, years & $43.78 \pm 11.84^{*}$ & $47.68 \pm 10.17$ & NS \\
Weight, kg & $74.26 \pm 9.64$ & $78.24 \pm 8.0$ & NS \\
Height, cm & $167.28 \pm 6.34$ & $169.24 \pm 6.14$ & NS \\
Operating time, min & $80: 20 \pm 19.51$ & $75: 24 \pm 29.27$ & NS \\
\hline
\end{tabular}

$*$ Values are mean $\pm \mathrm{SD}$.

pressed as the percent mean \pm standard deviation. Differences between LC and OC groups were determined by $\chi^{2}$ test for categorical variables and Student's unpaired t test for continuous scale variables; $\mathrm{p}$ values $<0.05$ were considered statistically significant.

\section{Results}

Both LC and OC patients had similar demographics (table 1). No postoperative liver failure or mortality occurred in any of the patients, and all were hemodynamically stable during the postoperative period. There was a significant increase in ALT and AST within the first $48 \mathrm{~h}$ postoperatively in LC patients compared with OC patients. In the first $48 \mathrm{~h}$ after the operation increased ALT was found in $26 / 50(52 \%)$ of LC patients but only $5 / 50$ $(10 \%)$ in OC patients. The difference was statistically significant ( $p<0.0001)$. Similarly a higher number of patients after LC had increased values in AST than after OC: 23 LC patients but only 6 OC patients. The difference was statistically significant $(p=0.0004)$. Total bilirubin, ALP, GGT and LDH levels remained unchanged from baseline in both groups with no significant difference between them (table 2, fig. 1).

\section{Discussion}

From the present study, it is clear that transient elevation of hepatic transaminase can occur after laparoscopic surgery. Pneumoperitoneum-induced hepatic hypoperfusion has been demonstrated in experimental and clinical studies. An intra-abdominal pressure of $15 \mathrm{~mm}$ $\mathrm{Hg}$ used in laparoscopy is higher than the normal portal blood pressure $(8 \mathrm{~mm} \mathrm{Hg})$. This pneumoperitoneum could therefore reduce portal flow and cause alteration in liver function. Hepatic perfusion is characterized by 
Fig. 1. The linear relationship in postoperative changes in ALT and AST between LC and OC.
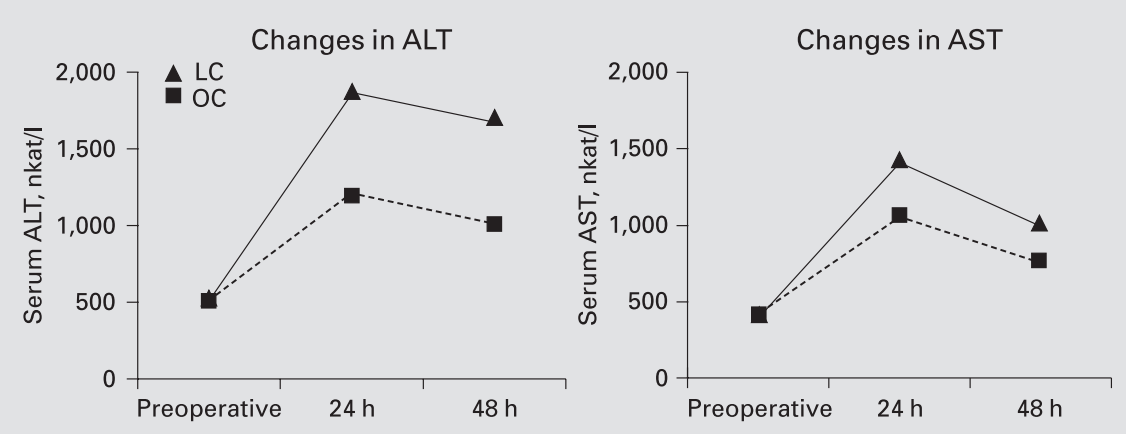

Table 2. Doubling of liver function tests from preoperative values to $48 \mathrm{~h}$ after operation in $\mathrm{LC}$ and $\mathrm{OC}$

\begin{tabular}{lrrrrrr}
\hline $\begin{array}{l}\text { Liver function } \\
\text { tests }\end{array}$ & \multicolumn{2}{l}{$\begin{array}{l}\text { LC patients } \\
(\mathrm{n}=50)\end{array}$} & & \multicolumn{2}{l}{$\begin{array}{l}\text { OC patients } \\
(\mathrm{n}=50)\end{array}$} & Value \\
\cline { 2 - 3 } & $\mathrm{n}$ & $\%$ & & $\mathrm{n}$ & $\%$ & \\
\hline ALT & 26 & 52 & & 5 & 10 & $0.0001^{*}$ \\
AST & 23 & 46 & & 6 & 12 & $0.0004^{*}$ \\
Total bilirubin & 9 & 18 & & 4 & 8 & $\mathrm{NS}$ \\
ALP & 2 & 4 & & 2 & $\mathrm{NS}$ \\
GGT & 0 & 0 & & 2 & 4 & $\mathrm{NS}$ \\
LDH & 2 & 1 & & 7 & 3.5 & $\mathrm{NS}$ \\
\hline
\end{tabular}

${ }^{*} \chi^{2}$-test; NS = not significant.

a unique autoregulatory mechanism, known as the hepatic arterial buffer response [12]. Richter et al. [13] reported that under conditions of $\mathrm{CO}_{2}$ pneumoperitoneum in the rat, hepatic arterial blood flow does not compensate for the intra-abdominal pressure-associated reduction of portal venous inflow, with the consequence of a marked decrease in total liver perfusion, inadequate oxygen supply and hepatocellular injury. Jakimowicz et al. [10] demonstrated a significant reduction of portal venous flow during LC, measured using a laser-Doppler technique.

Two mechanisms have been advanced to explain the elevation of ALT and AST after LC. One is hepatocellular damage [7-9] due to intra-abdominal hypertension-induced ischemic injury to the hepatocytes. This explanation is worrisome to a surgeon due to anticipation of an iatrogenic bile duct injury. The other mechanism is anesthesia-induced hepatic hypoperfusion. Transient liver dysfunction has been found to occur after many anesthetic agents [14-16]. But in the present study the same anesthesia protocols were used in both the LC and OC patients, therefore this was unlikely to have led to the enzyme changes we observed.

Pneumoperitoneum and its consequent intra-abdominal hypertension-induced hepatic ischemia appear more likely to be the cause. However, in patients with compromised liver function, these changes may be associated with adverse clinical effects. A low-pressure pneumoperitoneum or gasless abdominal wall lift approach should be considered in patients with compromised liver function.

\section{Conclusions}

Elevation of hepatic transaminases (ALT, AST) occurred after LC but were transient and clinically silent in patients with normal liver function. 


\section{References}

1 Cuschieri A: Laparoscopic cholecystectomy. J R Coll Surg Edinb 1999;44:187-190.

-2 Hasukić Š, Mešić D, Dizdarević E, Keser D, Hadžiselimović S, Bazardžanović M: Pulmonary function after laparoscopic and open cholecystectomy. Surg Endosc 2002;16:163-165.

-3 Koivusalo AM, Lindgren L: Effects of carbon dioxide pneumoperitoneum for laparoscopic cholecystectomy. Acta Anaesthesiol Scand 2000;44:834-841.

4 Clarke RS, Doggart JR, Lavery T: Changes in liver function after different types of surgery. Br J Anaesth 1976;48:119-128.

5 Hojjati M, Ghofrani M, Vala'i N: A quasi-experimental study on the effect of upper gastrointestinal surgery on liver function tests. Hepatogastroenterology 1998;45:1702-1705.

-6 Halevy A, Gold-Deutch R, Negri M, Lin G, Shlamkovich N, Evans S, Cotariu D, Scapa E, Bahar M, Sackier JM: Are elevated liver enzymes and bilirubin levels significant after laparoscopic cholecystectomy in the absence of bile duct injury? Ann Surg 1994;219:362364.
7 Morino M, Giraudo G, Festa V: Alterations in hepatic function during laparoscopic surgery: An experimental clinical study. Surg Endosc 1998;12:968-972.

8 Andrei VE, Schein M, Margolis M, Rucinski JC, Wise L: Liver enzymes are commonly elevated following laparoscopic cholecystectomy: Is elevated intra-abdominal pressure the cause? Dig Surg 1998;15:256-259.

-9 Saber AA, Laraja RD, Nalbandian HI, PablosMendez A, Hanna K: Changes in liver function tests after laparoscopic cholecystectomy: Not so rare, not always ominous. Am Surg 2000;66: 699-702.

10 Jakimowicz J, Stultiens G, Smulders F: Laparoscopic insufflation of the abdomen reduces portal venous flow. Surg Endosc 1998;12:129133.

11 Sato K, Kawamura T, Wakusawa R: Hepatic blood flow and function in elderly patients undergoing laparoscopic cholecystectomy. Anesth Analg 2000;90:1198-1202.
12 Lautt WW: Mechanism and role of intrinsic regulation of hepatic arterial blood flow: Hepatic arterial buffer response. Am J Physiol 1985;249:G549-G556.

13 Richter S, Olinger A, Hildebrandt U, Menger MD, Vollmar B: Loss of physiologic hepatic blood flow control ('hepatic arterial buffer response') during $\mathrm{CO}_{2}$-pneumoperitoneum in the rat. Anesth Analg 2001;93:872-877.

14 Gopal DV, Rosen HR: Abnormal findings on liver function tests. Interpreting results to narrow the diagnosis and establish a prognosis. Postgrad Med 2000;107(2):100-114.

-15 Augusti M, Elizalde I, Adalia R, Martinez-Palli G, Garcia-Valdecasas JC, Pique JM, Taura $\mathrm{P}$ : The effects of vasoactive drugs on hepatic blood flow changes induced by $\mathrm{CO}_{2}$ laparoscopy: An animal study. Anesth Analg 2001;93: 1121-1126.

16 Cray SH, Crawford MW, Khayyam N, Carmichael FJL: Effects of hypoxia and isoflurane on liver blood flow: The role of adenosine. $\mathrm{Br} \mathrm{J}$ Anaesth 2001;86:425-427. 